Résumés des conférences et travaux

\title{
Post-doctorats soutenus à la section pendant
} l'année 2013-2014

par ordre alphabétique des noms d'auteur

\section{(2) OpenEdition}

\section{Journals}

Édition électronique

URL : https://journals.openedition.org/ashp/1665

DOI : 10.4000/ashp.1665

ISSN : 1969-6310

Éditeur

Publications de l'École Pratique des Hautes Études

Édition imprimée

Date de publication : 1 septembre 2015

Pagination : $x \mathrm{XV}$

ISSN : 0766-0677

Référence électronique

"Post-doctorats soutenus à la section pendant l'année 2013-2014 », Annuaire de l'École pratique des hautes études (EPHE), Section des sciences historiques et philologiques [En ligne], $146 \mid 2015$, mis en ligne le 28 septembre 2015, consulté le 07 juillet 2021. URL : http://journals.openedition.org/ashp/1665 ; DOI : https://doi.org/10.4000/ashp.1665 


\section{DIPLÔMES DE L’ÉCOLE SOUTENUS À LA SECTION PENDANT L'ANNÉE 2013-2014}

par ordre alphabétique des noms d'auteur

"L'imagination éclairée par le goût ». Alfred Cortot pédagogue, d'après ses cours d'interprétation et ses commentaires des sonates de Beethoven, par M. Rémi JACOBS, sous la direction de $\mathrm{M}^{\mathrm{me}}$ Catherine MAssip, le 10 juin 2014.

\section{DOCTORATS SOUTENUS À LA SECTION PENDANT L'ANNÉE 2013-2014 par ordre alphabétique des noms d'auteur}

Études sur la morphosyntaxe du tabasaran littéraire, par $\mathrm{M}^{\mathrm{me}}$ Ayten BABALIYEva, sous la direction de M. Jean-Pierre MAHÉ, le 13 décembre 2013.

L'aide militaire française dans la politique de défense de la Côte d'Ivoire de 1960 à 2002, par M. Louis-Philippe BANGA, sous la direction de $\mathbf{M}^{m e}$ Nathalie GoEDERT et de M. Aka Kounmè, le $1^{\text {er }}$ février 2014.

Le «Bilad al-Zab» à l'époque médiévale. Étude historique et archéologique, par M. Ramzi Bedhiafi, sous la direction de M. François Deroche, le 29 mars 2014.

Lumière et mosquées en Égypte et Syrie médiévales, des conquêtes arabes (milieu du VII ${ }^{e}$ s.) à la fin de la dynastie ayyubide (milieu du XIII ${ }^{e}$ s.) : gestion de l'éclairage et portée symbolique, par $\mathrm{M}^{\mathrm{me}}$ Julie BONNERIC, sous la direction de M. JeanMichel Mouton, le 30 novembre 2013.

L'Opéra de Paris sous la direction d'André Messager et Leimistin Broussan (19081914), par $\mathrm{M}^{\mathrm{me}}$ Karine Boulanger, sous la direction de $\mathrm{M}^{\mathrm{me}}$ Catherine Massip, le 2 décembre 2013.

Une dynastie de libraires-imprimeurs de médecine à Paris : les d'Houry (1649-1790), par $\mathrm{M}^{\mathrm{me}}$ Anne BOYER, sous la direction de M. Frédéric BARBIER, le 22 mars 2014.

Révolution et nation entre le Portugal et l'Italie. Les relations politiques lusoitaliennes des Lumières à l'Internationale libérale de 1830, par M. Grégoire Bron, sous la direction de M. Gilles PÉcout, le 12 décembre 2013.

La vie des monuments de l'État. Histoire de la Caisse nationale des monuments historiques (1912-1978), par $\mathrm{M}^{\mathrm{me}}$ Valérie CARPENTIER, sous la direction de M. JeanMichel Leniaud, le 10 janvier 2014.

Idéologie royale iranienne au début de l'Islam, par $\mathrm{M}^{\mathrm{me}}$ Silvia Valentina Cazzato, sous la direction de M. Étienne DE LA VAISSIÈre, le 9 décembre 2013.

Une géographie historique du Campa du Sud. L'exemple du pays de Panrang (mi $X V I I I^{e}$ - début XXe siècle), par M. Fabien Chebaut-Me Mougamadou, sous la direction de M. Philippe PAPIN, le 26 novembre 2013. 
Éditer et commenter les Annales à la Renaissance: Marc-Antoine Muret lecteur de Tacite, par $\mathrm{M}^{\mathrm{me}}$ Lucie Claire, sous la direction de $\mathrm{M}^{\mathrm{me}}$ Perrine Galand, le 23 novembre 2013.

Genesi e sviluppo della critica del Cristianesimo nell'opera di Friedrich Nietzsche - Genèse et développement de la critique du christianisme dans l'œuvre de Friedrich Nietzsche, par M. Francesco D'Achille, sous la direction de M. GiUliano CAMPIONi et de M. JACQUes Le Rider, le 7 juillet 2014.

Michel Liénard (1810-1870), luxuriance et modestie de l'ornement au XIX siècle, par $\mathrm{M}^{\mathrm{me}}$ Sophie Derrot, sous la direction de M. Jean-Michel Leniaud, le 26 septembre 2014.

Les fortifications du Ladakh, de l'âge du Bronze à la perte d'indépendance (16831684 de n. è.), par M. Quentin Devers, sous la direction de M. Alain Thote, le 26 mars 2014.

La poésie de Paulin de Nole : des réseaux de communication du IV siècle aux bibliothèques médiévales de France et d'Italie - La poesia di Paolino di Nola : dalle reti di comunicazione del sec. IV alle biblioteche medioevali francesi ed italiane, par M. Franz Dolveck, sous la direction de M. Gian Carlo Alessio et de $\mathrm{M}^{\mathrm{me}}$ AnneMarie TurCAN-VERKERK, le 22 septembre 2014.

Théories et vocabulaire de la vision dans les mondes grec et latin du IVe au XII siècle, par $\mathrm{M}^{\mathrm{me}}$ Colette Dufossé, sous la direction de $\mathrm{M}^{\text {me }}$ Danielle JACQUART, le 7 décembre 2013.

Artigiano delle riforme. Stile rustico e ricerca della sapienza nell'opera di Bernard Palissy (1510-1590), par $\mathrm{M}^{\mathrm{me}}$ Juliette Ferdinand, sous la direction de M. Bernard Aikema et de $\mathrm{M}^{\mathrm{me}}$ Sabine Frommel, le 13 juin 2014.

Le théâtre des collèges parisiens au début du XVIe siècle. Textes et pratiques dramatiques, par M. Mathieu Ferrand, sous la direction de $\mathbf{M}^{\mathrm{me}}$ Perrine Galand, le 13 décembre 2013.

Le Grand Giral, Jean-Antoine Giral (1713-1787). L'architecte de l'embellissement du Peyrou de Montpellier, par M. Jean-Luc Fournet, sous la direction de $\mathrm{M}^{\mathrm{me}}$ Sabine Frommel, le 19 décembre 2013.

Étude du vocabulaire crétois d'après les inscriptions (époques archaïque, classique et hellénistique), par M. Gérard Genevrois, sous la direction de M. Laurent Dubois, le 18 juin 2014.

De la Théorie à la Praxis : réflexions sur les pratiques du dessin à l'Accademia di San Luca de Federico Zuccari à Carlo Maratti, par $\mathrm{M}^{\mathrm{me}}$ Rachel GeORGE, sous la direction de M. Michel Hochmann, le 17 décembre 2013.

Le temple d'Amenhotep III à Louqsor. Recueil d'inscriptions et commentaires, par M. Andras Gulyas, sous la direction de M. Pascal Vernus, le 8 février 2014.

La servitude pour dette au Campa du sud au XVIII siècle. Étude des archives légales $d u$ "Panduranga-Campa » de la Société asiatique de Paris, par $\mathrm{M}^{\mathrm{me}}$ Thérèse Guyot Becker, sous la direction de M. Philippe PAPIn, le 7 février 2014.

Le tombeau de Jean-Jacques Rousseau au Panthéon. Du mythe de la cabane rustique de Vitruve à sa fortune dans l'iconographie des traités d'architecture entre le XV et le XVIII siècle, par $\mathrm{M}^{\mathrm{me}}$ Eleonora Guzzo, sous la direction de M. Amedeo BELLUZZI et de $\mathrm{M}^{\mathrm{me}}$ Sabine Frommel, le 19 mai 2014. 
Art, artistes et commanditaires en Champagne du nord, milieu du XV $V^{e}$-fin du XVI siècle, par M. Maxence Hermant, sous la direction de M. Guy-Michel Leproux, le 14 décembre 2013.

Histoire du livre imprimé au Maroc. Aux origines d'une mutation au royaume chérifien (1865-1956), par M. Karim IfrAK, sous la direction de M. Frédéric BARBIER, le 16 janvier 2014.

La presse et les lettres : les épîtres paratextuelles et le programme éditorial de l'imprimeur Josse Bade (c. 1462-1535), par $\mathrm{M}^{\mathrm{me}}$ Louise Katz Simon, sous la direction de $\mathrm{M}^{\mathrm{me}}$ Perrine Galand, le 12 décembre 2013.

Les Archives de la princesse Iltani découvertes à Tell al-Rimah (XVIII siècle av. J.-C.), par $\mathrm{M}^{\mathrm{me}}$ Anne-Isabelle LANGLOIS, sous la direction de M. Dominique CHARPIN, le 11 octobre 2014.

Un village égyptien et sa campagne : étude de la microtoponymie du territoire d'Aphroditê $\left(V I^{e}-V I I I^{e} s\right.$.), par $\mathrm{M}^{\mathrm{me}}$ Isabelle MARTнот, sous la direction de M. Jean-Luc FOURNET, le 6 décembre 2013.

Du statère au sesterce. Rome et la Gaule septentrionale et orientale (150 av. J.-C. 50 apr. J.-C.), par M. Stéphane Martin, sous la direction de M. Michel ReddÉ et M. Michel Amandry, le 30 novembre 2013.

Medicina e potere : Angelo Gatti e l'inoculazione del vaiolo (1724-1798), par $\mathrm{M}^{\mathrm{me}}$ Veronica Massai, sous la direction de M. Daniele Menozzi et de M. JeanClaude WAQUET, le 10 septembre 2014.

Lire Homère à la Renaissance. Philologie humaniste et tradition grecque. Sur les traces de Vettor Fausto et de Guillaume Budé, par M. Patrick Morantin, sous la direction de $\mathrm{M}^{\mathrm{me}}$ Brigitte Mondrain, le 14 décembre 2013.

Les émirs dans le sultanat mamelouk sous les sultans Barquq et Farag (784-815/13821412), par M. Clément Onimus, sous la direction de M. Jean-Michel Mouton, le 10 décembre 2013.

"Unterm reinsten Himmel der unsicherste Boden ». Die Gro£stadterfahrung in Goethes Italiendichtung - "Sous le ciel le plus pur, le terrain le plus incertain ». L'expérience de la grande ville dans les textes du Voyage en Italie, par M. Malte Osterloh, sous la direction de M. Michael JAEger et de M. Jacques Le Rider, le 17 mai 2014.

Ragusan Patronage of Italian Renaissance Painting, par M. Kiril PENUSLISKI, sous la direction de M. Michel Hochmann, le 19 décembre 2013.

Le petit prophète. Leonardo Lessio SJ tra controversie teologiche e santita (15541623), par $\mathrm{M}^{\mathrm{me}}$ Eleonora RAI, sous la direction de M. Jean-Louis QUANTIN et de $\mathrm{M}^{\mathrm{me}}$ Paola Vismara, le 20 mars 2014.

Gênes et Rome 1590-1650 : pratiques culturelles et échanges artistiques, par $\mathrm{M}^{\mathrm{me}}$ Anne Rivoallan, sous la direction de M. Michel Hochmann, le 9 décembre 2013.

Les modes de construction au Maghreb médiéval d'après la pratique et les textes juridiques, par M. Lotfi SaAdaoui, sous la direction de M. François Deroche, le 29 mars 2014.

Les dépôts de fondation dans l'Égypte ancienne, par M. François SchmitT, sous la direction de M. Pascal Vernus, le 21 décembre 2013. 
La distribution spatiale de l'habitat en Chine dans la plaine Centrale à la transition entre le Néolithique et l'âge du Bronze (env. 2500-1050 av. J.-C.), par M ${ }^{\mathrm{me}}$ Pauline Sebillaud, sous la direction de M. Alain Thote et de M. Lixin Wang, le 2 juin 2014.

Style. Théorie et pratique dans l'œuvre de Viollet-le-Duc, par M. Andrea SERRAU, sous la direction de M. Jean-Michel Leniaud et de M. Giovanni LeOni, le 26 mai 2014.

Les Vues de Rome de Giovanni Antonio Dosio (1550-1570). La représentation de l'Antiquité dans la ville moderne, par $\mathrm{M}^{\mathrm{me}}$ Agnieszka WiATRZYK, sous la direction de $\mathrm{M}^{\mathrm{me}}$ Claudia Conforti et de $\mathrm{M}^{\mathrm{me}}$ Sabine Frommel, le 29 septembre 2014.

Formation des paradigmes religieux entre essai et hagiographie. Étude de deux Biji du bouddhisme Chan des Song du Sud (1127-1279), par M. Chao ZHANG, sous la

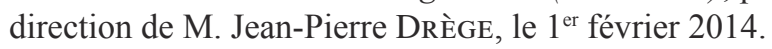

\section{POST-DOCTORATS SOUTENUS À LA SECTION PENDANT L'ANNÉE 2013-2014}

par ordre alphabétique des noms d'auteur

L'Église de France et ses emblèmes (1802-1940). Héraldique, sigillographie, paramentique, prosopographie des évêques en France et en quelques territoires rattachés, par M. Christophe Rousseau-Lefebvre, sous la direction de M. Michel Pastoureau, le 19 novembre 2013.

\section{HABILITATIONS À LA DIRECTION DE RECHERCHES SOUTENUES À LA SECTION PENDANT L'ANNÉE 2013-2014} par ordre alphabétique des noms d'auteur

La " construction de l'unité civique » dans l'Italie républicaine, par $\mathrm{M}^{\mathrm{me}}$ Clara BERRENDONNER, sous la direction de M. Jean-Louis Ferrary, le 23 novembre 2013.

Culture, médias et patrimoines : enjeux contemporains, par $\mathrm{M}^{\mathrm{me}}$ Agnès CALlu, sous la direction de M. Jean-Michel Leniaud, le 7 mai 2014.

Entre sphère privée et res publica. Droit, cité et société à Rome, par M. Julien Dubouloz, sous la direction de M. Jean-Louis Ferrary, le 30 novembre 2013.

Écrire l'histoire de l'architecture et des arts plastiques à Lyon aux XIXe et XXe siècles, par M. Philippe DufieuX, sous la direction de M. Jean-Michel Leniaud, le 4 juin 2014. 
Maîtres des écoles et auteurs spirituels du Moyen Âge latin : histoire des textes et histoire des doctrines (XII ${ }^{e}-X V^{e}$ siècles), par M. Cédric GIRAUD, sous la direction de M. Patrick Henriet, le 28 juin 2014.

Sciences, techniques et pratiques visuelles dans l'Antiquité tardive et à Byzance, par M. Stavros LAZARIs, sous la direction de $\mathrm{M}^{\mathrm{me}}$ Danielle JACQUART, le 17 juin 2014.

Poétiques latines et néo-latines du sommeil, par $\mathrm{M}^{\mathrm{me}}$ Virginie Leroux, sous la direction de $\mathrm{M}^{\mathrm{me}}$ Perrine Galand, le 3 décembre 2013.

Autour de Plotin. Métaphysique et platonisme, par M. Sylvain Roux, sous la direction de M. Philippe Hoffmann, le 30 novembre 2013. 